Pesq. Vet. Bras. 37(4):313-318, abril 2017 DOI: $10.1590 /$ S0100-736X2017000400003

\title{
Intoxicação natural por Senecio brasiliensis em equinos ${ }^{1}$
}

\author{
Welden Panziera ${ }^{2 *}$, Ronaldo M. Bianchi², Renata D. Mazaro ${ }^{3}$, Paula R. Giaretta ${ }^{2}$, \\ Gabriele B. Silva ${ }^{4}$, Diego R.P. Silva ${ }^{4}$ e Rafael A. Fighera ${ }^{5}$
}

\begin{abstract}
Panziera W., Bianchi R.M., Mazaro R.D., Giaretta P.R., Silva G.B., Silva D.R.P. \& Fighera R.A. 2017. [Natural poisoning by Senecio brasiliensis in horses.] Intoxicação natural por Senecio brasiliensis em equinos. Pesquisa Veterinária Brasileira 37(4):313-318. Departamento de Patologia, Universidade Federal de Santa Maria, Av. Roraima 1000, Santa Maria, RS 97105-900, Brazil. E-mail: weldenpanziera@yahoo.com.br

Poisoning due to plants of the genus Senecio is considered an important cause of death in livestock. Senecio brasiliensis is the most common species involved in spontaneous cases. This paper describes the epidemiological, clinical and pathological aspects of a natural outbreak of intoxication with Senecio brasiliensis affecting two out of eighteen horses in a farm. The cases occurred in the municipality of Sao Martinho da Serra, Rio Grande do Sul State, Southern Brazil. A large amount of Senecio brasiliensis in its sprouting stage was found in the fields where the horses used to be kept. A four-year-old, Crioulo mare and a five-month-old, male, Crioulo foal were affected. The clinical course was acute in the mare, comprising three days and chronic in the foal, lasting for two months. The main clinical signs in both animals included apathy, anorexia, weight loss, dehydration and neurological signs. Moreover, subcutaneous edema was noted in the ventral abdomen and the mucous membranes were icteric in the mare and pale in the foal. The serum biochemistry in the foal showed hypoalbuminemia $(2.1 \mathrm{~g} / \mathrm{dL})$, increased activity of the serum alkaline phosphatase $(434 \mathrm{U} / \mathrm{L})$ and gamma glutamyl transferase $(119 \mathrm{U} / \mathrm{L})$. In the hemogram, a mild normocytic normochromic anemia was noted in the foal (Hct: 30\%; MCV: 39.5 fL; MCH: 15.3pg). Necropsy findings observed in both horses included an enlarged, dark red and firm liver with extensive areas of hemorrhage and enhanced lobular pattern on cut surface. Histologically, the liver showed variable degrees of fibrosis, coagulative necrosis, biliary ducts hyperplasia, megalocytosis and cholestasis. In the brain, the astrocytes in the cerebrum were degenerated and similar to Alzheimer type II astrocytes. The diagnosis in this outbreak was established based on the epidemiology, clinical picture, gross and histologic findings. It is noteworthy that the coagulative necrosis, minimal fibrosis, and biliary duct hyperplasia observed in the mare are characteristic of a subacute presentation. On the other hand, the fibrosis that was more prominent in the foal characterizes a chronic hepatopathy.
\end{abstract}

INDEX TERMS: Equine diseases, hepatotoxic plants, Asteraceae, Senecio brasiliensis, pyrrolizidine alkaloids, pathology, poisonous plants, plant poisoning.

\footnotetext{
${ }^{1}$ Recebido em 15 de abril de 2016.

Aceito para publicação em 9 de novembro de 2016.

${ }^{2}$ Laboratório de Patologia Veterinária (LPV), Centro de Ciências Rurais (CCR), Universidade Federal de Santa Maria (UFSM), Av. Roraima 1000, Bairro Camobi, Santa Maria, RS 97105-900, Brasil. *Autor para correspondência: weldenpanziera@yahoo.com.br

${ }^{3}$ Curso de Medicina Veterinária, CCR-UFSM, Av. Roraima 1000, Camobi, Santa Maria, RS 97105-900, Brasil. Bolsista PIBIC/CNPq/UFSM.

${ }^{4}$ Clínica de Equinos, Hospital Veterinário Universitário (HVU), UFSM, Av. Roraima 1000, Camobi, Santa Maria, RS 97105-900, Brasil.

${ }^{5}$ Departamento de Patologia, Centro de Ciências da Saúde (CCS), UFSM, Av. Roraima 1000, Camobi, Santa Maria, RS 97105-900, Brasil.
}

RESUMO.- Intoxicações por plantas do gênero Senecio representam uma importante causa de morte em animais de produção, sendo Senecio brasiliensis a espécie mais envolvida nos casos de intoxicação espontânea. Nesse trabalho, são descritos os aspectos epidemiológicos, clínicos e anatomopatológicos de um surto de intoxicação natural por $\mathrm{Se}$ necio brasiliensis em equinos. Dois equinos, de um total de dezoito, foram afetados. Os casos ocorreram em uma propriedade rural no município de São Martinho da Serra, Rio Grande do Sul, Brasil. Grande quantidade de exemplares de Senecio brasiliensis em estágio de brotação foi encontrada 
na área onde os equinos estavam. Os animais eram da raça Crioula, sendo uma égua com quatro anos e um potro com cinco meses. A égua apresentou evolução aguda da doença com duração de três dias e o potro evolução crônica de dois meses. As principais manifestações clínicas observadas em ambos os equinos incluíam apatia, anorexia, emagrecimento, desidratação e sinais neurológicos. Além disso, havia moderado edema subcutâneo na região ventral do abdômen e marcada icterícia nas mucosas ocular, oral e vulvar da égua e palidez das mucosas no potro. $\mathrm{Na}$ avaliação bioquímica do potro, havia acentuado aumento da atividade sérica da gamaglutamil transferase (119 U/L), aumento da fosfatase alcalina (434 U/L) e hipoalbuminemia $(2,1 \mathrm{~g} / \mathrm{dL})$. No hemograma do mesmo, observou-se leve anemia normocítica e normocrômica (30\% de hematócrito; VCM: 39,5 fL; HCM: 15,3pg). As alterações presentes em ambas as necropsias foram observadas principalmente no fígado, que estava difusamente aumentado de tamanho, escuro e firme. Na superfície de corte, observaram-se acentuação do padrão lobular e hemorragia. Histologicamente, no fígado dos equinos, havia graus variados de necrose coagulativa, hemorragia, fibrose, proliferação de ductos biliares, hepatomegalocitose e bilestase. Nas diferentes seções analisadas do encéfalo, havia alterações astrocitárias restritas à substância cinzenta, predominantemente, no córtex telencefálico. Os astrócitos degenerados eram similares aos astrócitos de Alzheimer tipo II. 0 diagnóstico da intoxicação nesses casos foi baseado na epidemiologia, no quadro clínico, nas lesões de necropsia e nos achados histopatológicos. Cabe ressaltar que a necrose de coagulação e a discreta fibrose e proliferação de ductos biliares observados na égua, constituiu uma apresentação subaguda da intoxicação, enquanto que a fibrose, mais predominante no potro, caracterizou uma hepatopatia crônica.

TERMOS DE INDEXAÇÃO: Doenças de equinos, plantas hepatotóxicas, Asteraceae, Senecio brasiliensis, alcaloides pirrolizidínicos, patologia, plantas tóxicas, intoxicação por plantas.

\section{INTRODUÇÃO}

Intoxicações por plantas do gênero Senecio representam uma importante causa de morte em animais de produção, principalmente na espécie bovina, que resultam em grandes perdas econômicas na cadeia produtiva (Tokarnia et al. 2012). No Rio Grande do Sul, as principais espécies de Senecio associadas com intoxicações em animais de produção incluem: S. brasiliensis, S. oxyphyllus, S. cisplatinus, S. heterotrichius, S. selloi, S. leptolobus, S. tweediei e S. madagascariensis; a espécie $S$. brasiliensis é a mais envolvida nos casos de intoxicação natural (Méndez 1991, Méndez \& Riet-Correa 2008, Tokarnia et al. 2012). A toxicidade das espécies de Senecio deve-se à presença de alcaloides que pertencem ao grupo das pirrolizidinas, os quais são hepatotóxicos e produzem lesão crônica e irreversível, caracterizada pela inibição da mitose de hepatócitos (Radostits et al. 2007, Méndez \& Riet-Correa 2008, Tokarnia et al. 2012).

Casos de intoxicação por Senecio spp. em equinos são menos frequentes do que em bovinos, embora estas sejam consideradas duas das espécies mais suscetíveis à ação da planta (Radostits et al. 2007, Tokarnia et al. 2012). Há pou- cos casos de intoxicação natural (Carvalho \& Maugé 1946, Curial \& Guimarães 1958, Gava \& Barros 1997) e experimental (Pilati \& Barros 2007) em equinos no Brasil, e à semelhança do que ocorre em bovinos, a doença nos equinos geralmente apresenta um curso clínico crônico, com o aparecimento dos primeiros sinais clínicos semanas ou meses após a ingestão da planta. Menos comumente pode haver casos de intoxicação aguda (Tokarnia et al. 2012). As principais manifestações clínicas observadas nos equinos incluem distúrbios neurológicos, apatia, anorexia, disfagia, emagrecimento, edema subcutâneo e icterícia (Gava \& Barros 1997, Riet-Correa et al. 1998, Pilati \& Barros 2007, Tokarnia et al. 2012). Além de bovinos e equinos, há descrições esporádicas de intoxicação natural (Ilha et al. 2001, Grecco et al. 2011, Giaretta et al. 2014) e experimental (Barros et al. 1989) por Senecio spp. em ovinos, espontânea em búfalos (Corrêa et al. 2008) e induzidas experimentalmente em caprinos e frangos (Tokarnia et al. 2012).

0 objetivo deste trabalho é relatar um surto de intoxicação natural por Senecio brasiliensis em equinos na região Sul do Brasil, abordando os aspectos epidemiológicos, clínicos e anatomopatológicos dessa condição.

\section{MATERIAL E MÉTODOS}

Os dados epidemiológicos e clínicos foram obtidos durante três visitas à propriedade onde os casos ocorreram. Amostra de sangue do potro afetado foi coletada para a realização de hemograma e testes de função hepática (gamaglutamil transferase [GGT], aspartato aminotransferase [AST], fosfatase alcalina [AP]). Durante as visitas realizou-se a necropsia de dois equinos e fragmentos de órgãos foram coletados, fixados em formol tamponado a $10 \%$, processados rotineiramente para histologia e corados pela técnica de hematoxilina e eosina (HE). Espécimes da planta observados no campo foram coletados durante uma das visitas e enviados para classificação no Departamento de Botânica da Universidade Federal de Santa Maria.

\section{RESULTADOS}

O material botânico enviado foi classificado como Senecio brasiliensis. Os casos ocorreram no período de abril a junho de 2014, em uma pequena propriedade rural do município de São Martinho da Serra, na região central do Estado do Rio Grande do Sul. Na propriedade havia dezoito equinos da raça Crioula, sendo 10 éguas (idades entre 4 a 12 anos), seis potros (idades entre 4 a 7 meses) e dois equinos machos (idades de 5 e 8 anos). Os equinos eram mantidos em campo nativo, com moderada oferta de gramíneas misturadas à grande quantidade de Senecio brasiliensis em estágio de brotação. Os animais estavam nessa área há aproximadamente oito meses antes do início do surto. Nenhuma outra planta tóxica conhecida foi observada no campo. Dois equinos dos dezoito citados anteriormente adoeceram, sendo uma égua com quatro anos e um potro com cinco meses. De modo geral, os dois animais demonstraram sinais clínicos semelhantes, entretanto cursos clínicos distintos. A égua morreu em abril e apresentou um curso clínico agudo da doença, com duração de três dias. Já a duração dos sinais clínicos do potro prosseguiu por cerca de dois meses após a morte da égua, o que caracterizou uma apresentação crônica. 
As manifestações clínicas observadas em ambos os equinos incluíam apatia, anorexia, emagrecimento (mais acentuado no potro devido à cronicidade), desidratação e sinais neurológicos, caracterizados por incoordenação, andar a esmo, compressão da cabeça contra objetos e decúbito esternal e lateral. Além disso, havia moderado edema subcutâneo na região ventral do abdômen e marcada icterícia nas mucosas ocular, oral e vulvar da égua, sinais clínicos não observados no potro. No potro, observou-se palidez das mucosas. É importante ressaltar que ambos os equinos demonstraram início das manifestações simultaneamente, entretanto, o potro persistiu com os sinais por um longo período, com episódios de melhora e piora temporária do quadro clínico. Além disso, nenhum outro equino da propriedade adoeceu.

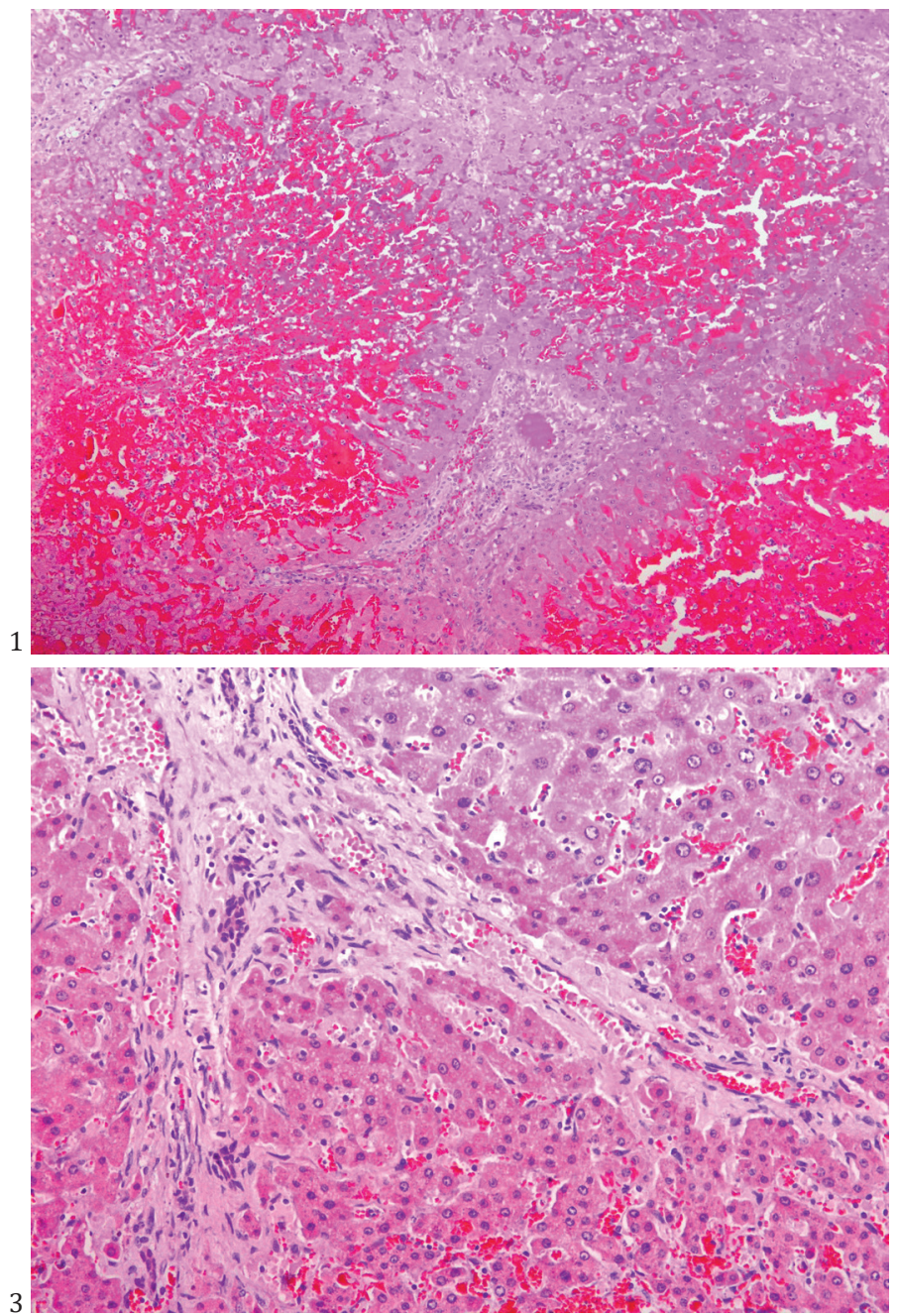

Fig.1. Intoxicação natural por Senecio brasiliensis em equinos. Fígado da égua. Marcada hemorragia e necrose de hepatócitos que se estende das regiões centrolobulares dos lóbulos para as regiões mediozonais. Nota-se que há preservação de hepatócitos das regiões periportais. HE, obj.20x.

Fig.3. Intoxicação natural por Senecio brasiliensis em equinos. Fígado do potro. Fibrose periportal moderada associada à leve proliferação de ductos biliares. Observa-se que o tecido fibroso disseca os cordões de hepatócitos adjacentes. HE, obj.20x.
$\mathrm{Na}$ avaliação bioquímica do potro, havia acentuado aumento da atividade sérica da gamaglutamil transferase (GGT) (119 U/L, valor de referência [VR]: 7-17 U/L \{Thrall et al. 2012\}), aumento nos níveis da fosfatase alcalina (434 U/L; VR: 90-290 U/L [Thrall et al. 2012]) e hipoalbuminemia (2,1g/dL; VR: 2,7-3,7g/dL [Thrall et al. 2012]). No hemograma, observou-se somente leve anemia normocítica e normocrômica (30\% de hematócrito, VR: 32-53\%; VCM: 39,5 fL, VR: 37-59 fL; HCM: 15,3 pg, VR: 12-20pg \{Grondin \& Dewitt 2010\}). Os demais parâmetros hematológicos e bioquímicos avaliados estavam dentro dos limites normais. Amostra de sangue da égua não foi coletada porque o animal já havia morrido quando o proprietário solicitou a visita à propriedade.

Na necropsia, o fígado dos equinos estava moderada-

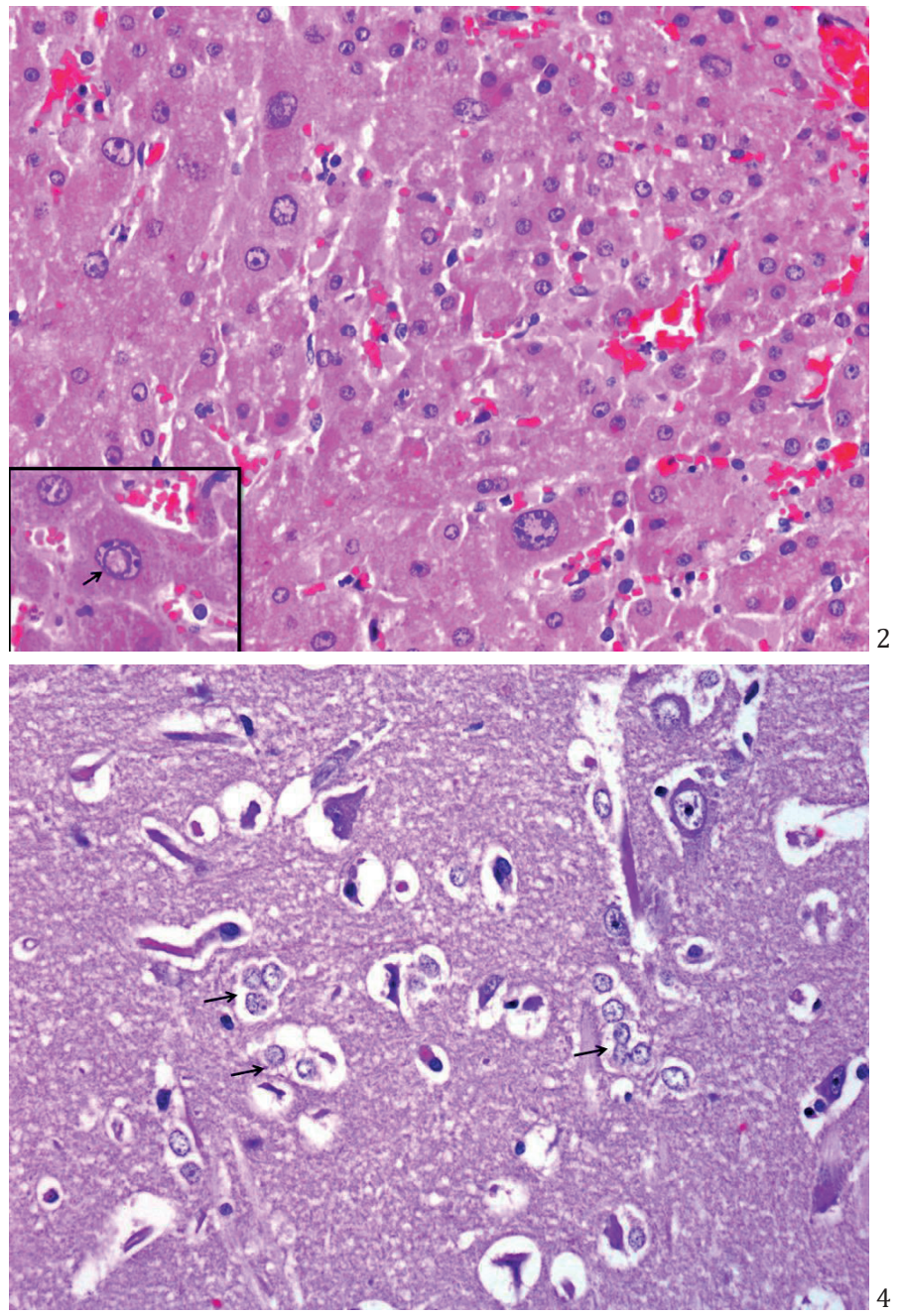

Fig.2. Intoxicação natural por Senecio brasiliensis em equinos. Fígado do potro. Numerosos hepatócitos com o núcleo acentuadamente aumentado de tamanho (megalocitose). No detalhe (canto inferior esquerdo) hepatócito com corpúsculo de inclusão intranuclear eosinofílico (pseudoinclusão [seta]). HE, obj.40x.

Fig.4. Intoxicação natural por Senecio brasiliensis em equinos. Encéfalo do potro (córtex telencefálico). Astrócitos com o núcleo vesicular e com a cromatina marginalizada (astrócitos de Alzheimer tipo II). Observa-se que essas células estão aos pares ou formam pequenos grupos (setas). HE, obj.40x. 
mente aumentado de tamanho (hepatomegalia difusa), escuro e firme. Na superfície de corte, observaram-se marcada acentuação do padrão lobular e hemorragia, caracterizado por áreas vermelho-escuras intercaladas com áreas mais claras. Esta representou a principal alteração macroscópica comum para os dois equinos. Outros achados macroscópicos observados somente na égua incluíam estado corporal regular, edema subcutâneo ventro-abdominal, acentuada icterícia das mucosas, do tecido subcutâneo e tecido adiposo da cavidade abdominal e hemorragias no omento e na serosa dos órgãos do trato gastrointestinal. No potro, além da alteração hepática, havia palidez das mucosas e estado corporal ruim.

Histologicamente, no fígado dos equinos, observaram-se graus variados de necrose coagulativa, hemorragia, fibrose, proliferação de ductos biliares e hepatomegalocitose. A hemorragia e a necrose foram mais acentuadas no fígado da égua e se estendiam das regiões centrolobulares dos lóbulos para as regiões mediozonais (Fig.1), sendo que em algumas áreas a necrose tornava-se massiva. Havia também áreas com bilestase. A fibrose, proliferação de ductos biliares e hepatomegalocitose eram leves no fígado da égua, denotando um aspecto subagudo da intoxicação. Já os achados histológicos observados no fígado do potro eram crônicos e caracterizavam-se principalmente pela marcada hepatomegalocitose (Fig.2) e presença de maior quantidade de tecido fibroso e proliferação de ductos biliares (Fig.3), quando comparado à égua. No interior do núcleo de numerosos hepatócitos havia glóbulos eosinofílicos ovais, caracterizando pseudoinclusões (Figura 3 [detalhe no canto inferior esquerdo]).

Nas diferentes seções analisadas do encéfalo de ambos os equinos, observaram-se alterações astrocitárias restritas à substância cinzenta, predominantemente, no córtex telencefálico. Estes astrócitos geralmente encontravam-se aos pares e ocasionalmente formando pequenos grupos, continham núcleos aumentados de tamanho, vesiculares (vazios), com a cromatina marginada e, ocasionalmente, com nucléolos proeminentes. Essas células eram similares aos astrócitos de Alzheimer tipo II (Fig.4). Nos demais órgãos dos dois equinos não foram observados alterações.

\section{DISCUSSÃO}

A epidemiologia, o quadro clínico, as lesões macroscópicas e os achados histopatológicos observados nos equinos deste surto são idênticos aos descritos por outros autores (Carvalho \& Maugé 1946, Curial \& Guimarães 1958, Gava \& Barros 1997, Pilati \& Barros 2007, Tokarnia et al. 2012) para intoxicação por Senecio spp. em equinos e constituem evidências que permitem o diagnóstico desta condição.

Estudos sobre causas de morte ou razões para eutanásia em equinos demonstram a baixa prevalência ou ausência de casos de intoxicação por Senecio spp. nesta espécie (Karam et al. 2004, Pierezan et al. 2009, Pimentel et al. 2009, Lucena et al. 2010, Marcolongo-Pereira et al. 2014). No Rio Grande do Sul, a ocorrência de intoxicação por Senecio spp. em equinos parece ser muito baixa se comparada com a intoxicação em bovinos, apesar de a espécie ser comprovadamente suscetível a intoxicação experimental por $S$. brasiliensis (Pilati \& Barros 2007). Estima-se que bovinos e equinos são cerca de 30 a 40 vezes mais suscetíveis à ação dos alcaloides pirrolizidínicos (APs), quando comparados aos ovinos e caprinos. A diferença parece estar relacionada à capacidade desses pequenos ruminantes detoxificarem os APs no fígado, provavelmente devido à dieta consumida antes da domesticação. Além disso, ovinos e caprinos são menos seletivos quanto à ingestão de alimentos, desenvolvendo, em virtude disso, resistência às toxinas (Radostits et al. 2007, Santos et al. 2008).

Possíveis explicações para a baixa ocorrência da intoxicação em equinos seriam o pastejo mais seletivo dessa espécie, menor palatabilidade da planta para esses animais ou a suplementação dos equinos com concentrados no período de menor disponibilidade de pastagens (Gava \& Barros 1997, Tokarnia et al. 2012). No presente caso, sugere-se que a baixa disponibilidade de gramíneas, associada à ausência de suplementação e um aumento na lotação da área possam ter contribuído para a ingestão do $S$. brasiliensis. Outro fator importante é o tamanho da planta que estava em estágio de brotação. Apesar dos equinos possuírem seletividade, as plantas pequenas podem ter sido facilmente ingeridas durante o pastejo.

Os surtos de intoxicação por plantas do gênero Senecio têm sido observados em todas as estações do ano e geralmente são de caráter crônico, tanto em ruminantes quanto em equinos (Tokarnia et al. 2012). Nos casos crônicos, os animais ingerem quantidades não suficientes para induzir a forma aguda, mas ocorrem danos hepáticos que serão manifestados posteriormente (Lucena et al. 2010). Nos equinos deste surto foram observadas lesões hepáticas de caráter subagudo e crônico, representando uma manifestação atípica. Esses animais apresentaram cursos clínicos e achados anatomopatológicos diferentes, o que permitiu caracterizar as intoxicações. Além disso, o histórico também auxiliou sobremaneira na caracterização. Acredita-se que a égua ingeriu uma quantidade diária maior da planta durante o pastejo e o potro pequenas quantidades. Essa hipótese justificaria a morte mais precoce da égua e a mais tardia do potro e reforça o efeito acumulativo que as plantas desse gênero têm quando ingeridas em pequenas quantidades diárias por períodos prolongados (Tokarnia et al. 2012).

Clinicamente os equinos deste surto apresentaram manifestações típicas de intoxicação por Senecio spp., à semelhança da literatura (Carvalho \& Maugé 1946, Curial \& Guimarães 1958, Gava \& Barros 1997, Riet-Correa et al. 1998, Pilati \& Barros 2007, Tokarnia et al. 2012). Esses sinais incluíam principalmente distúrbios neurológicos, apatia, anorexia, emagrecimento, edema subcutâneo, icterícia e anemia. A duração do quadro clínico de três dias até a morte da égua e um curso prolongado de dois meses no potro, representam respectivamente, achados clínicos agudos e crônicos. Todas estas manifestações estão estritamente relacionadas com a insuficiência hepática e dependem da quantidade da planta ingerida, assim como o intervalo de tempo (Pilati \& Barros 2007, Lucena et al. 2010, Tokarnia et al. 2012).

Os sinais clínicos neurológicos observados nos equinos desse caso são decorrentes da encefalopatia hepática e resultam do acúmulo na corrente sanguínea, no líquido cefa- 
lorraquidiano e no encéfalo de substâncias como a amônia, ácidos graxos de cadeias curtas e mercaptanos, além de alterações nas concentrações de neurotransmissores. Normalmente, as substâncias tóxicas são eliminadas quando ocorre sua passagem pelo fígado, o que não acontece quando há lesão hepática difusa e grave, culminando em insuficiência hepática. Consequentemente, essas substâncias podem chegar ao encéfalo e, como falsos neurotransmissores provocam vários sinais clínicos neurológicos. A amônia é considerada a principal substância envolvida na patogênese da encefalopatia hepática (Summers et al. 1995, Barros 2010, Tokarnia et al. 2012).

A icterícia e o edema subcutâneo observados somente na égua desse surto são secundários à insuficiência hepática e caracterizam um achado clínico agudo. A icterícia ocorre devido a um defeito do fígado na excreção e secreção de pigmentos, ácidos biliares e sais biliares, com consequente deposição dessas substâncias nos tecidos. Sugere-se também que a icterícia possa ser decorrente da hemólise intravascular que ocorre por causas não bem esclarecidas em equinos com insuficiência hepática (Pilati \& Barros 2007, Santos et al. 2008). 0 edema subcutâneo é oriundo da hipoproteinemia, sendo atribuído à falha na síntese de albumina e outras proteínas do plasma pelo fígado (Santos et al. 2008). 0 emagrecimento mais acentuado no potro e a anemia prolongada, também representam sinais de insuficiência hepática, entretanto de caráter crônico, e denotam a característica prolongada da intoxicação. Embora os demais sinais clínicos, como apatia e anorexia, sejam inespecíficos, eles auxiliam na suspeita clínica do comprometimento hepático.

As lesões macroscópicas e histológicas da intoxicação por Senecio brasiliensis observadas nos equinos desse surto são características e semelhantes às descrições na literatura para plantas do gênero Senecio (Gava \& Barros 1997, Pilati \& Barros 2007, Tokarnia et al. 2012) e, quando associadas aos dados epidemiológicos, permitem o diagnóstico definitivo da intoxicação. Alterações hepáticas representam o principal achado macroscópico visualizado nos equinos acometidos pela doença. 0 fígado dos animais apresenta-se mais escuro, firme e com marcada acentuação do padrão lobular (Barros 2010, Tokarnia et al. 2012). Lesões idênticas foram observadas no fígado de ambos os equinos necropsiados deste surto. As demais lesões extra-hepáticas, que incluem principalmente icterícia, hemorragias e edema subcutâneo e cavitários, também ocorrem com frequência (Gava \& Barros 1997, Pilati \& Barros 2007) e devem ser levadas em consideração, pois representam sinais de insuficiência hepática.

Na intoxicação natural em equinos, os principais achados histopatológicos consistem de fibrose hepática, principalmente periportal, hepatomegalocitose e hiperplasia biliar, porque geralmente pequenas quantidades da planta são ingeridas por períodos mais prolongados (Gava \& Barros 1997, Pilati \& Barros 2007). Já a característica das lesões histológicas observadas nos equinos intoxicados experimentalmente e que ingerem quantidades maiores da planta em períodos curtos é diferente. Essas lesões caracterizam-se principalmente pela marcada necrose coagulativa e hemorragias que variam de centrolobular a massiva. Em- bora os casos descritos neste trabalho sejam de intoxicação natural, as lesões histológicas observadas no fígado da égua eram de caráter subagudo e semelhante às descritas anteriormente para os casos de intoxicação experimental. A necrose e a hemorragia representam a principal característica dos casos de intoxicação mais aguda, diferentemente do padrão de lesão observada nos casos de intoxicação crônica, em que se observam principalmente fibrose, proliferação de ductos e hepatomegalocitose acentuada, como se evidenciou no potro desse surto. Outra característica histológica importante, mais observada nos casos crônicos, é a presença de pseudoinclusões. A visualização dessas estruturas auxilia no diagnóstico (Barros 2010).

Testes de função hepática demonstraram alterações no nível sérico de atividade da GGT e fosfatase alcalina no potro, devido à colestase. A GGT é uma enzima originária das membranas dos canalículos e ductos biliares e o aumento da sua atividade sérica está relacionada exclusivamente à colestase intra e extra-hepática e a proliferação de ductos biliares. Esta enzima normalmente está restrita ao retículo endoplasmático liso, onde o sistema de oxidase mista é ativo. Os alcaloides pirrolizidínicos são ativados por este sistema, causando lesões nos hepatócitos e liberação da GGT no soro (Santos et al. 2008). Consequentemente, a determinação da atividade sérica da GGT representa um dos melhores parâmetros de diagnóstico bioquímico para intoxicação por Senecio spp. em equinos e bovinos, uma vez que a atividade da fosfatase alcalina tem um grande intervalo de referência nessas espécies (Barros et al. 2007, Santos et al. 2008, Bain 2011). Entretanto, testes de função hepática são geralmente pouco confiáveis para detectar casos subclínicos da intoxicação, pois a função hepática é normal nessa fase (Barros et al. 2007). Com isso, a biopsia hepática pode ser utilizada como um importante método que auxilia no diagnóstico de lesões hepáticas difusas, pois é uma técnica com alto valor prognóstico e permite a detecção de animais acometidos ou não pela intoxicação (Barros et al. 2007, Pilati \& Barros 2007).

Deve-se incluir no diagnóstico diferencial de intoxicação por Senecio spp. em equinos outras plantas que causam lesões hepáticas, principalmente aquelas que contêm pirrolizidinas como princípio tóxico (Tokarnia et al. 2012), ou plantas que causam alterações hepáticas agudas, como Trema micrantha (Bandarra et al. 2010, Tokarnia et al. 2012, Pavarini et al. 2013). Outras doenças que podem cursar com quadros neurológicos também são diagnósticos diferenciais, e incluem principalmente raiva, encefalomielites virais e mieloencefalite por protozoário (Santos et al. 2008, Lucena et al. 2009, Barros 2010, Tokarnia et al. 2012). Portanto, clínicos que trabalham com a espécie equina devem considerar intoxicação por Senecio spp. como diagnóstico diferencial em equinos que apresentarem sinais de insuficiência hepática e a avaliação epidemiológica e os achados anatomopatológicos são importantes para o diagnóstico preciso dessa toxicose.

\section{REFERÊNCIAS}

Bain P.J. 2011. Liver, p.211-230. In: Latimer K.S. (Ed.), Duncan and Prasse's Veterinary Laboratory Medicine: clinical pathology. 5th ed. Wiley-Blackwell, Ames. 509p. 
Bandarra P.M., Pavarini S.P., Raymundo D.L., Corrêa A.M.R., Pedroso P.M.O. \& Driemeier D. 2010. Trema micrantha toxicity in horses in Brazil. Equine Vet. J. 42(5):456-459.

Barros C.S.L., Metzdorf L.L., Santos M.N., Barros S.S. \& Peixoto P.V. 1989. Intoxicação experimental por Senecio brasiliensis (Compositae) em ovinos. Pesq. Vet. Bras. 9(3/4):55-67.

Barros C.S.L., Castilhos L.M.L., Rissi D.R., Kommers G.D. \& Rech R.R. 2007. Biópsia hepática no diagnóstico da intoxicação por Senecio brasiliensis (Asteraceae) em bovinos. Pesq. Vet. Bras. 27(1):53-60.

Barros C.S.L. 2010. Fígado vias biliares e pâncreas exócrino, p.183-290. In: Santos R.L. \& Alessi A.C. (Eds), Patologia Veterinária. Roca, São Paulo. 892p.

Carvalho G.S.T. \& Maugé G.C. 1946. Ação tóxica de Senecio brasiliensis Lessing, fam. Compositae. Revta Fac. Med. Vet. S. Paulo 3:131-136.

Corrêa A.M.R., Bezerra P.S.J., Pavarini S.P., Santos A.S., Sonne L., Zlotowski P., Gomes G. \& Driemeier D. 2008. Senecio brasiliensis (Asteraceae) poisoning in Murrah buffaloes in Rio Grande do Sul. Pesq. Vet. Bras. 28(3):187-189.

Curial O. \& Guimarães J.P. 1958. Cirrose hepática enzoótica no cavalo. Mem. Inst. Oswaldo Cruz 56:635-643.

Gava A. \& Barros C.S.L. 1997. Senecio spp. poisoning in horses in southern Brazil. Pesq. Vet. Bras. 17(1):36-40.

Giaretta P.R., Panziera W., Hammerschmitt M.E., Bianchi R.M., Galiza G.J.N., Wiethan I.S., Bazzi T. \& Barros C.S.L. 2014. Clinical and pathological aspects of chronic Senecio spp. poisoning in sheep. Pesq. Vet. Bras. 34(10):967-973.

Grecco F.B., Estima-Silva P., Marcolongo-Pereira C., Soares M.P., Collares G. \& Schild A.L. 2011. Seneciose crônica em ovinos no sul do Rio Grande do Sul. Pesq. Vet. Bras. 31(4):326-330.

Grondin T.M. \& Dewitt S. 2010. Normal hematology of the horse and donkey, p.821-828. In: Weiss D.J. \& Wardrop K.J. (Eds), Schalm's Veterinary Hematology. 6th ed. Wiley-Blackwell, Ames. 1206p.

Ilha M.R.S., Loretti A.P., Barros S.S. \& Barros C.S.L. 2001. Intoxicação espontânea por Senecio brasiliensis (Asteraceae) em ovinos no Rio Grande do Sul. Pesq. Vet. Bras. 21(3):123-138.

Karam F.S.C., Soares M.P., Haraguchi M., Riet-Correa F., Méndez M.C. \& Jarenkow J.A. 2004. Aspectos epidemiológicos da seneciose na região sul do Rio Grande do Sul. Pesq. Vet. Bras. 24(4):191-198.

Lucena R.B., Rissi D.R., Maia L.A., Flores M.M., Dantas A.F.A., Nobre V.M.T., Riet-Correa F. \& Barros C.S.L. 2010. Intoxicação por alcaloides pirrolizidínicos em ruminantes e equinos no Brasil. Pesq. Vet. Bras. 30(5):447452.
Marcolongo-Pereira C., Estima-Silva P., Soares M.P., Sallis E.S.V., Grecco F.B., Raffi M.B., Fernandes C.G. \& Schild A.L. 2014. Doenças de equinos na região Sul do Rio Grande do Sul. Pesq. Vet. Bras. 34(3):205-210.

Méndez M.C. 1991. Intoxicações por plantas e micotoxinas hepatotóxicas, p.43-102. In: Riet-Correa F., Méndez M.C. \& Schild A.L. (Eds), Intoxicações por Plantas e Micotoxicoses em Animais Domésticos. Vl.1. Editorial Agropecuaria Hemisferio Sur S.R.L., Montevideo. 340p.

Méndez M.C. \& Riet Correa F. 2008. Plantas que causam fibrose hepática, p.25-32. In: Ibid. (Eds), Plantas Tóxicas e Micotoxicoses. Editora Universitária/UFPEL , Pelotas. 298p.

Pavarini S.P., Bandinelli M.B., Bassuino D.M., Correa G.L.F., Bandarra P.M., Driemeier D., Hohendorff R.V. \& Both M.C. 2013. Novos aspectos sobre a intoxicação por Trema micrantha (Cannabaceae) em equídeos. Pesq. Vet. Bras. 33(11):1339-1344.

Pierezan F., Rissi D.R., Rech R.R., Fighera R.A., Brum J.S. \& Barros C.S.L. 2009. Achados de necropsia relacionados com a morte de 335 equinos: 1968-2007. Pesq. Vet. Bras. 29(3):275-280.

Pilati C. \& Barros C.S.L. 2007. Intoxicação experimental por Senecio brasiliensis (Asteraceae) em equinos. Pesq. Vet. Bras. 27(7):287-296.

Pimentel L.A., Oliveira D.M., Galiza G.J.N., Rego R.O., Dantas A.F.M. \& Riet-Correa F. 2009. Doenças do sistema nervoso central de equídeos no semi-árido. Pesq. Vet. Bras. 29(7):589-597.

Radostits O.M., Gay C.C., Hinchcliff K.W. \& Constable P.D. 2007. Diseases associated with toxins in plants, fungi, cyanobacteria, plant-associated bacteria, and venoms in ticks and vertebrate animals, p.1850-1920. In: Ibid (Eds.), Veterinary medicine: A textbook of the diseases of cattle, horses, sheep, pigs and goats. 10th ed. Saunders Elsevier, Philadelphia. 2156p.

Riet-Correa F., Soares M.P. \& Méndez M.C. 1998. Intoxicações em eqüinos no Brasil. Ciência Rural 28(4):715-722.

Santos J.C.A., Riet-Correa F., Simões S.V.D. \& Barros C.S.L. 2008. Patogênese, sinais clínicos e patologia das doenças causadas por plantas hepatotóxicas em ruminantes e equinos no Brasil. Pesq. Vet. Bras. 28(1):1-14.

Summers B.A., Cummings J.F. \& De Lahunta A. 1995. Degenerative diseases of the central nervous system, p.208-214. In: Ibid. (Eds), Veterinary Neuropathology. Mosby, St Louis. 525p.

Thrall M.A., Weiser G., Allison R.W. \& Campbell T.W. 2012. Clinical case presentation, p.657. In: Ibid. (Eds), Veterinary Hematology and Clinical Chemistry. 2nd ed. Wiley-Blackwell, Ames. 762p.

Tokarnia C.H., Brito M.F., Barbosa J.D., Peixoto P.V. \& Döbereiner J. 2012. Plantas/micotoxinas que afetam o fígado, p.147-204. In: Ibid. (Eds), Plantas Tóxicas do Brasil para Animais de Produção. 2ª ed. Helianthus, Rio de Janeiro. 586p. 